\title{
Effect of External Magnetic Field on Long DC Arc Characteristics with Rng-shaped Anode
}

\author{
Akio Hashizawa ${ }^{1}$, Manabu Tanaka ${ }^{1}$, Takayuki Watanabe ${ }^{1}$, and Tomohiro Koga ${ }^{2}$ \\ ${ }^{1}$ Department of chemical engineering, Kyushu University, 744 Motooka, Nishi-ku, Fukuoka, 819-0395, Japan \\ ${ }^{2}$ Clean Technology Co. Ltd., 3209-1 Hineno, Izumisano, Osaka 598-0021, Japan
}

\begin{abstract}
Fluctuation phenomena of plasma jet flow in an innovative long DC arc system with a ring-shaped anode were successfully clarified by a high-speed camera visualization. The long DC arc with long electrode gap distance more than $350 \mathrm{~mm}$ has been applied to gas decomposition due to its advantages of long plasma length, resulting in long residence time of treated gas. However, large heat loss at a conventional hemispherical-shaped anode was a critical issue in the long DC arc system. Therefore, the ring-shaped anode was utilized to convert large energy loss at the anode into the plasma jet flow. High-speed camera observation revealed the effect of external magnetic field on the fluctuation phenomena. Plasma jet fluctuates with the frequency of several tens $\mathrm{Hz}$ when the magnetic field was applied. These understanding of arc and plasma jet fluctuation enables to improve the capability of long DC arc system.
\end{abstract}

\section{Introduction}

Thermal plasmas have been received a great attention in applications to waste treatment. This is due to their unique advantages in the waste decomposition such as high temperature and abundant radicals, which promote the decomposition and conversion of waste into environmentally benign materials in a compact system (Heberlein and Murphy, 2008). Thermal plasmas over $10,000 \mathrm{~K}$ can destroy any kind of non-degradable material without the generation of undesired chemical compounds such as furan, dioxin, sulfur oxide, and nitrogen oxide.

A long DC arc is one of most attractive thermal plasma sources. The long DC arc can be generated between a cathode rod and a hemi-spherical anode with a longer gap distance than $300 \mathrm{~mm}$. This is one order longer than conventional DC arcs. Therefore, the residence time of the treated material is sufficiently long. Numerical (Choi et al., 2013) and experimental studies (Li et al., 2012) have been reported and feasibility of long DC arc process for organic waste decomposition was revealed (Li et al., 2013). Effect of external magnetic field on the arc fluctuation have also been clarified.

In order to improve the energy efficiency of the long DC arc processes, a ring-shaped anode was successfully applied to convert the energy loss at the conventional hemi-spherical anode to the available energy as thermal plasma jet generated downstream from the anode (Tanaka et al., 2018). Fluctuation characteristics of the long DC arc and the plasma jet flow have been clarified on the basis of the high-speed visualization. However, the fluctuation characteristics of the long DC arc with external magnetic field have not been studied yet, although these understandings are essential to expand the capability of the long DC arc system in industry.

The purpose of the present work is to investigate the correlation between the arc and plasma jet fluctuations. High-speed camera observation with synchronized voltage measurements was carried out to understand the effect of the external magnetic field on the arc and plasma jet fluctuation.

\section{Experimental Methods}

Schematic diagram of long DC arc system is shown in Figure 1. Arc plasma in the DC torch generally consists of two plasma regions. One is arc discharge region, where Joule heating occurs. The arc channel was connected between a cylindrical cathode and the anode. Another is the plasma jet region generated below the anode nozzle structure due to the strong drag gas flow. The long DC arc was generated between a cathode and an anode with their gap distance of $350 \mathrm{~mm}$. The rod-shaped cathode was utilized with a length of $160 \mathrm{~mm}$ and a diameter of $20 \mathrm{~mm}$. On the other hand, the ring-shaped copper with $20 \mathrm{~mm}$ in a thickness, $100 \mathrm{~mm}$ in an outer diameter, and $15 \mathrm{~mm}$ in nozzle diameter was utilized as the ring-shaped anode. Plasma jet is generated in the downstream region of the ring-shaped anode in the long DC arc torch. An auxiliary metal wire plays a role of a pilot electrode for an ignition

* Corresponding author: watanabe@ chem-eng.kyushu-u.ac.jp 


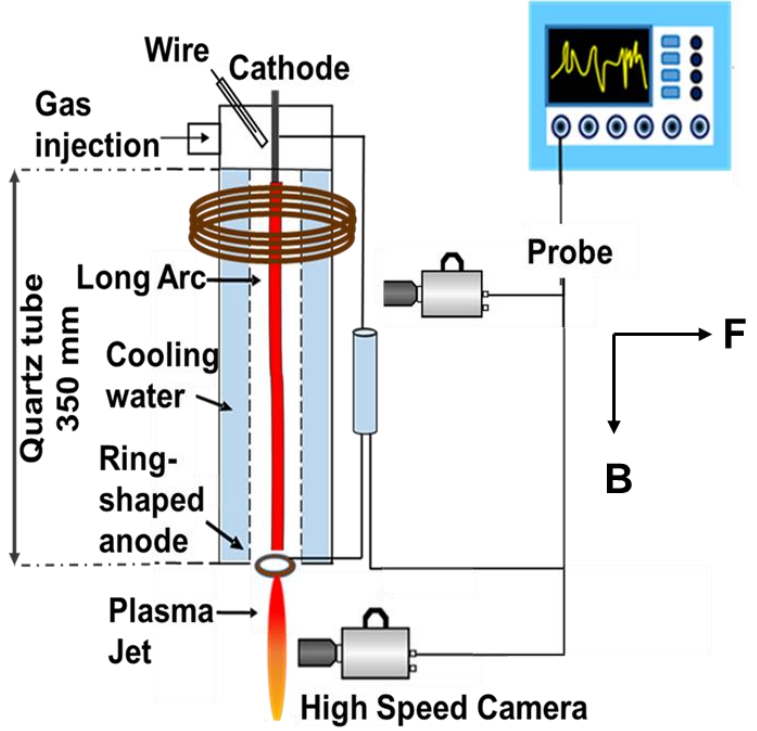

Figure 1. Experimental set up of long DC arc system.

of the long DC arc because a breakdown between the electrodes with long gap distance of $350 \mathrm{~mm}$ under atmospheric pressure is difficult. This wire was temporally contacted with the anode at the moment of gas breakdown. Then, the wire was pulled back toward the cathode. Finally, the arc was transferred from the wire to the cathode.

The generated long DC arc was confined by an inner quartz tube which was cooled by circulating water. The inner diameter of the plasma confinement tube was 60 $\mathrm{mm}$. Nitrogen as plasma forming gas at flow rate of 30 $\mathrm{L} / \mathrm{min}$ was injected from top of the system. An arc current was fixed at $10 \mathrm{~A}$, arc voltage was in the range from 600 to $1000 \mathrm{~V}$. The arc power was then in the range of 6 to 10 $\mathrm{kW}$.

The coil was placed at $80 \mathrm{~mm}$ from the cathode. Coil current was changed in the range from $0-1.0 \mathrm{~A}$ to investigate the effect of external magnetic field on arc and plasma jet fluctuation phenomena.

Arc and plasma jet fluctuation were visualized by two high-speed cameras (FASTCAM, Photron and Miro 110, Phantom). Framerate of these cameras were changed from $10^{3}$ to $10^{4} \mathrm{fps}$. Shutter exposed time were $10^{-4} \mathrm{~s}$. These cameras were synchronized with oscilloscope (Scope Corder DL850, Yokogawa) to understand arc and plasma jet fluctuation.

\section{Results and Discussion}

\subsection{Arc fluctuation}

The effect of the coil current on arc fluctuation is presented in this section. High-speed snapshots of the arc at different coil currents are shown in Figure 2. Obtained snapshots shows that the long DC arc column was destabilized under the conditions with the external magnetic field. The existence probability distribution of the arc was estimated based on the binarization and its summation of the high-speed snapshots. Figure 3 shows the counter maps of arc existence probabilities at different coil currents. The probability of arc was defined by the ratio of the time during which the arc existed to total measured time. Therefore, unity means that the arc always exits and zero means the arc does not exist. Increase of the coil currents clearly leads to the spread arc region.

The direction of the main arc path and the magnetic field are the same, therefore, the external magnetic field does not have effective influences on the arc. However, the obtained results indicated the external magnetic field strongly affects to the arc motion. This originates from the partial axisymmetric of the arc column. While the axial current flow $j_{\mathrm{z}}$ is dominant, horizontal component $j_{\mathrm{r}}$ or $j_{\theta}$ of arc current exists. Once the Lorenz force acts on the arc due to its horizontal component of the arc current, the Lorenz force becomes larger owing to the bending of the arc. As a result, the arc existence area is expanded.

Arc voltage waveform at different coil currents are shown in Figure 4. Mean arc voltage and its fluctuation with the external magnetic field was obviously larger than

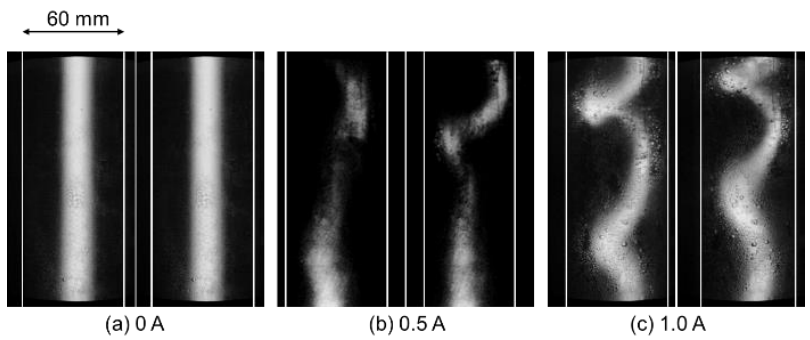

Figure 2. High-speed snap shots of arc at different coil current. (a) $0 \mathrm{~A}$, (b) $0.5 \mathrm{~A}$, and (c) $1.0 \mathrm{~A}$.

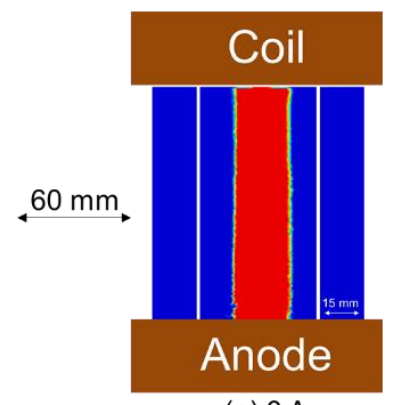

(a) $0 \mathrm{~A}$

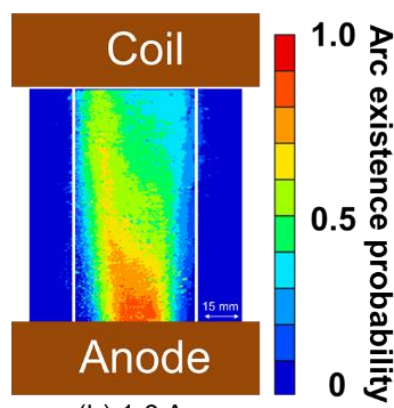

(b) $1.0 \mathrm{~A}$
Figure 3. Arc existence probability with coil current of (a) $0 \mathrm{~A}$ and (b) $1.0 \mathrm{~A}$.
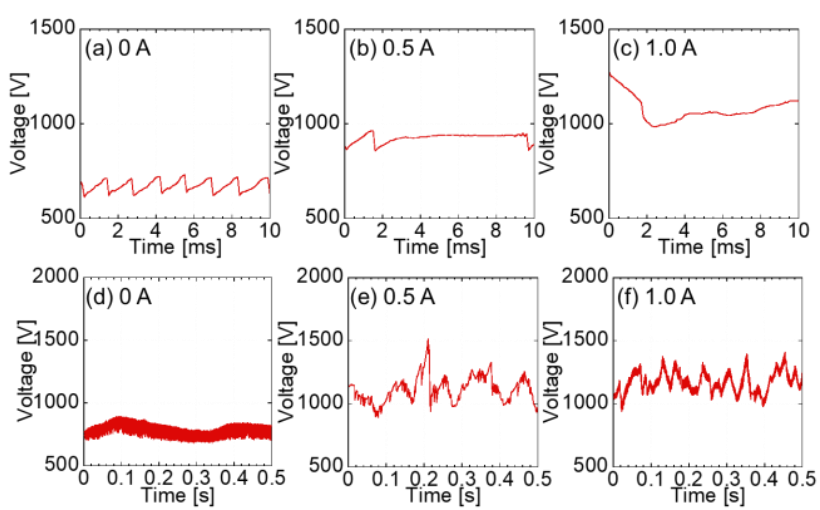

Figure 4. Arc voltage waveform at different coil current. Short time span with (a) $0 \mathrm{~A}$, (b) $0.5 \mathrm{~A}$, and (c) 1.0 A. Long time span with (d) $0 \mathrm{~A}$, (e) $0.5 \mathrm{~A}$, and (f) $1.0 \mathrm{~A}$. 

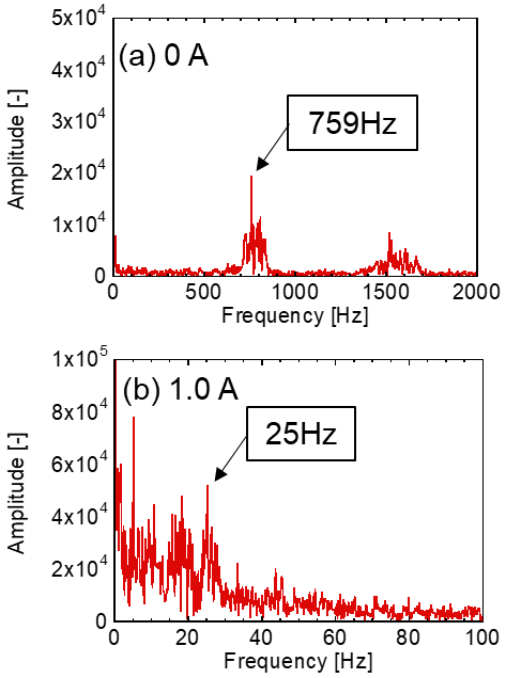

Figure 5. FFT analysis on arc voltage fluctuation with coil current of (a) $0 \mathrm{~A}$ and (b) $1.0 \mathrm{~A}$.

that without the magnetic field. This is due to the longer arc length, which originates from the arc swirl motion.

The arc fluctuation frequencies at different coil currents were characterized through the Fast Fourier Transform (FFT) as shown in Figure 5. The frequency without magnetic field was about $700 \mathrm{~Hz}$. This is attributed to the arc restrike motion, which is one of the most important phenomena in a non-transferred torch (Duan et al., 2002). On the other hand, the characteristics frequency was about $25 \mathrm{~Hz}$ when the external magnetic field was applied. This can be considered as the arc rotation due to the Lorentz force from the high-speed snapshots.

\subsection{Plasma jet fluctuation}

The effect of the coil current on plasma jet fluctuation is presented in this section. High-speed snapshots of plasma jet at different coil currents are shown in Figure 6. Time variation of the luminance areas of the plasma jet at different coil currents are shown in Figure 7, which were estimated from the high-speed snapshots. The luminance area fluctuation with the magnetic field was much larger than that without the magnetic field. This can be explained by the voltage fluctuation due to the arc motion. The voltage fluctuation with the magnetic field was larger than that without the magnetic field, as shown in Figure 4. Larger voltage fluctuation led to larger fluctuation of arc power, resulting in the larger fluctuation of the plasma jet area.

The counter maps of existence probabilities of the plasma jet at different coil currents are presented in Figure 8. The definition of the existence probability of the plasma jet was the same with the arc existence probability, which was mentioned in the previous section. Plasma jet area at unity of the existence probability becomes smaller with an increase of the coil currents. This is due to the larger plasma jet fluctuation. In contrast, the whole plasma jet area becomes larger when the coil current increase from 0 A to $0.5 \mathrm{~A}$. Then, that becomes lower when the coil

current increase from $0.5 \mathrm{~A}$ to $1.0 \mathrm{~A}$. This trend can be explained by two factors.

First factor is the arc power, which is increased with the increase of the coil current because the arc voltage increased due to the Lorentz force. Higher arc power simply leads to larger plasma jet area. Another factor is

(a) $0 \mathrm{~A}$
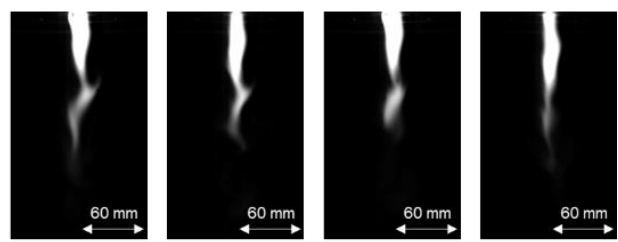

(b) $0.5 \mathrm{~A}$
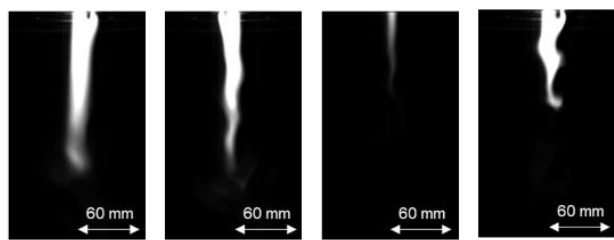

(c) $1.0 \mathrm{~A}$
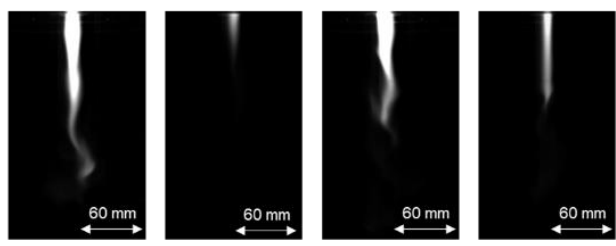

Figure 6. Snap shots of plasma jet at different coil current. (a) $0 \mathrm{~A}$, (b) $0.5 \mathrm{~A}$, and (c) $1.0 \mathrm{~A}$.
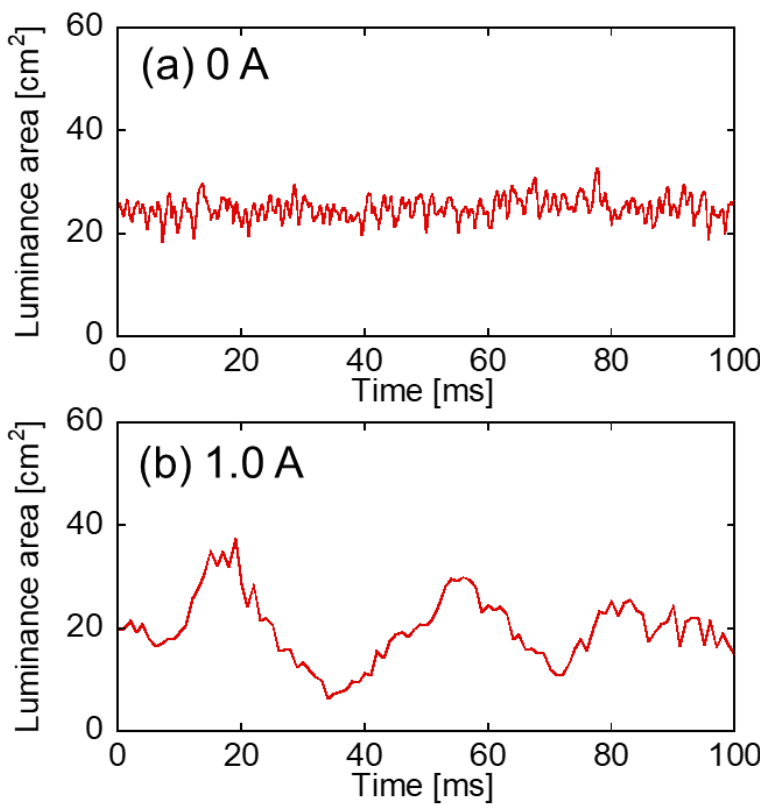

Figure 7. Fluctuation of luminance area of plasma jet with coil current of (a) $0 \mathrm{~A}$ and (b) $1.0 \mathrm{~A}$.

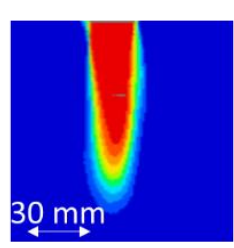

(a) $0 \mathrm{~A}$

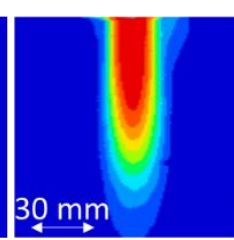

(b) $0.5 \mathrm{~A}$

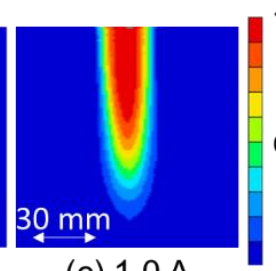

(c) $1.0 \mathrm{~A}$

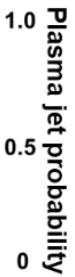

$5 \frac{\frac{0}{0}}{\frac{0}{3}}$

Figure 8. Plasma jet existence probability at different coil current. (a) $0 \mathrm{~A}$, (b) $0.5 \mathrm{~A}$, and (c) $1.0 \mathrm{~A}$. 
the momentum balance between the axial motion and swirl motion. Momentum in swirl direction increase with the increase of the coil current. Larger momentum in swirl direction leads to the entrainment of the cold surrounding air at the outside region of the anode nozzle. This leads to the smaller plasma jet area when the coil current increases. Plasma jet area at $0.5 \mathrm{~A}$ of the coil current becomes maximum because of these two factors.

Fluctuation characteristics of the plasma jet was discussed on the basis of FFT analysis of the luminance area fluctuation of the plasma jet, which was divided into two regions, upstream and downstream. Fluctuation characteristics of the plasma jet in the upstream region at different coil currents were summarized in Figure 9 (a), (b), and (c). The plasma jet frequency at $759 \mathrm{~Hz}$ was the similar with the arc fluctuation due to the restrike phenomena when the magnetic field was not applied. On the other hand, the plasma jet frequency at several tens $\mathrm{Hz}$ was the same with the arc fluctuation due to the arc swirl motion when the magnetic field was applied.

The plasma jet fluctuations at the downstream region at different coil currents were also summarized in Figure 9 (d), (e), and (f). The plasma jet frequency in the downstream region with the magnetic field was similar to the arc fluctuation mentioned above. This is due to the stronger momentum in swirl direction when the magnetic field was applied. On the other hand, the characteristic frequency of the plasma jet at the downstream without magnetic field was found at $388 \mathrm{~Hz}$. This indicates that the characteristic frequency of the plasma jet fluctuation at the downstream region was different from the arc fluctuation frequency when no magnetic field was applied. In the fringe of the plasma jet flow, a number of eddies periodically forms due to the entrainment of the cold surrounding air, which phenomena was firstly reported by Pfender et al. (1991). In recent, such eddy formation has been reproduced by numerical simulation (Shigeta, 2016). These results suggested that the plasma jet fluctuation with the characteristic frequency of several hundred $\mathrm{Hz}$ possibly occurs due to the eddy formation. Furthermore, these fluctuations were observed only at the downstream region. The observed plasma jet fluctuation without magnetic field is attributed to the eddy formation due to the entrainment of the surrounding air.

\section{Conclusion}

The long DC arc system with the ring-shaped anode was successfully established. The two high-speed cameras observation synchronized with the voltage measurement enabled to understand effect of coil current on fluctuation characteristics of the arc and the plasma jet.

Strong correlation between the arc fluctuation and the plasma jet fluctuation was found in the cases with and without the external magnetic field. Higher coil currents for the external magnetic field leads to larger arc fluctuation, resulting in the larger plasma jet fluctuation. This plasma jet fluctuation frequency was several tens $\mathrm{Hz}$

\section{Upstream}

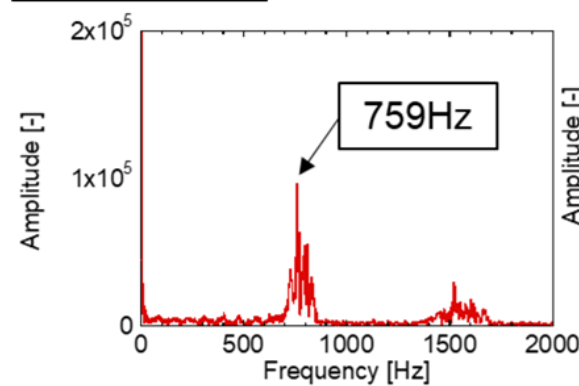

(a) $0 \mathrm{~A}$

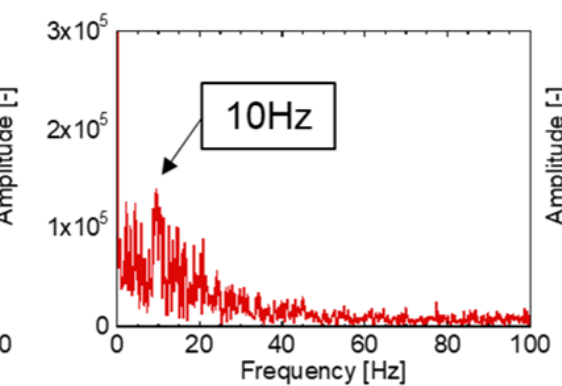

(b) $0.5 \mathrm{~A}$

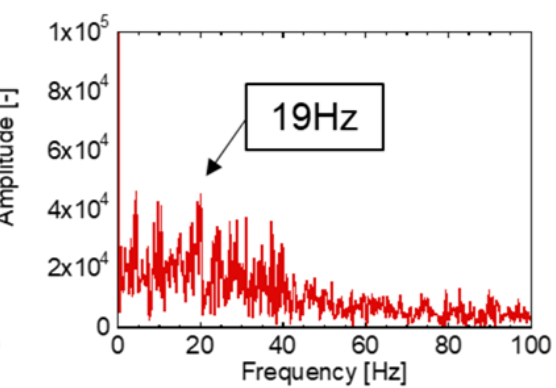

(c) $1.0 \mathrm{~A}$

\section{Downstream}

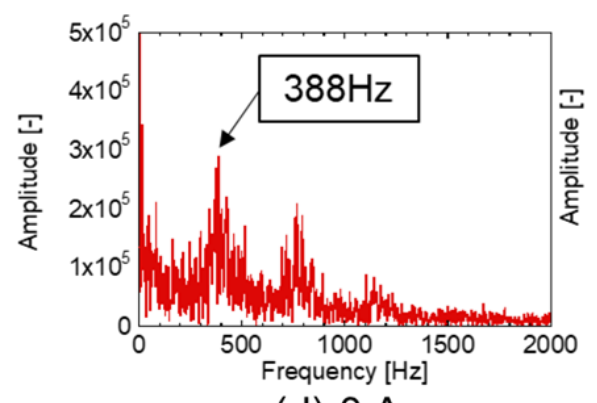

(d) $0 \mathrm{~A}$

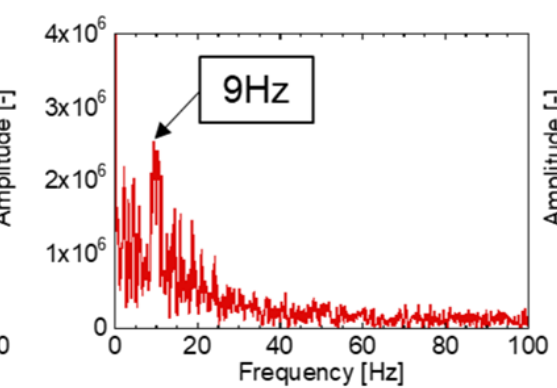

(e) $0.5 \mathrm{~A}$

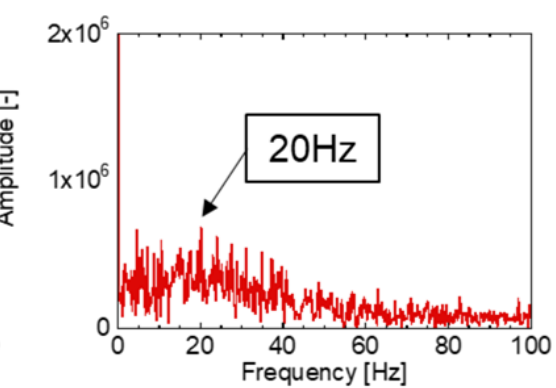

(f) $1.0 \mathrm{~A}$

Figure 9. FFT analysis on upstream and downstream plasma jet fluctuation at different coil current. Upstream region with (a) $0 \mathrm{~A}$, (b) $0.5 \mathrm{~A}$, and (c) $1.0 \mathrm{~A}$. Downstream region with (d) $0 \mathrm{~A}$, (e) $0.5 \mathrm{~A}$, and (f) $1.0 \mathrm{~A}$. 
and is attributed to the swirl motion of the arc. On the other hand, characteristic frequency of the plasma jet fluctuation at about $700 \mathrm{~Hz}$ due to the arc restrike phenomena and that at about $400 \mathrm{~Hz}$ due to the entrainment of the surrounding cold air were dominant when no external magnetic field was applied.

These understanding of the fundamental phenomena in the long DC arc system with external magnetic field enable to utilize this higher temperature heat source consisting of the discharge region and the plasma jet region for various innovative processing.

\section{References}

Choi, S., T. Li, T. Watanabe, T. Nakayama, and K. Otsuki; "Numerical Analysis of Temperature Distribution in the Long DC Arc Thermal Plasma for Waste Treatment," Journal of Chemical Engineering of Japan, 46, 201-208 (2013)

Duan, Z., and J. Heberlein; "Arc Instabilities in a Plasma Spray Torch," Journal of Thermal Spray Technology, 44, 44-51 (2002)

Heberlein, J. and A. B. Murphy; "Thermal Plasma Waste Treatment", Journal of Physics D: Applied Physics, 41, 053001 (2008)

Li, T., S. Choi, T. Watanabe, T. Nakayama, and T. Tanaka; "Discharge and Optical Characteristics of Long Arc Plasma of Direct Current Discharge," Thin Solid Films, 523, $72-75$ (2012)

Li, T., T. Watanabe, K. Ochi, and K. Otsuki; "Liquid Waste Decomposition by Long DC Arc under Atmospheric Pressure," Chemical Engineering Journal, 231, 155-162 (2013).

Pfender, E., J. Fincke, and R. Spores; "Entrainment of Cold Gas into Thermal Plasma Jets," Plasma Chemistry and Plasma Processing, 11, 529-543 (1991).

Shigeta, M.; "Turbulence Modeling of Thermal Plasma Fows," Journal of Physics D: Applied Physics, 49, 493001 (2016)

Tanaka, M., T. Watanabe, H. Soeda, and T. Koga; "Plasma Jet Characteristics in Long DC Arc with Ring-shaped Anode", Journal of Fluid Science and Technology, 13, JFST0027 (2018). 